\title{
Is There an Increasing Incidence of Gastroesophageal Junctional Adenocarcinoma and Barrett Esophagus in Asia? A Review of Diagnostic Conundrums
}

\author{
Phei Oon Tan ${ }^{a, b}$ Alex Yu Sen Soh ${ }^{c}$ Chika Kusano $^{d}$ Yeong Yeh Lee ${ }^{a, e}$ \\ Takuji Gotoda ${ }^{f}$
}

${ }^{a}$ GI Function \& Motility Unit, Hospital Universiti Sains Malaysia, Kota Bharu, Malaysia; ${ }^{b}$ Gastroenterology Unit, Department of Medicine, Hospital Raja Permaisuri Bainun, Ipoh, Malaysia; 'Division of Gastroenterology and Hepatology, Department of Medicine, National University Hospital, Singapore, Singapore; ${ }^{\mathrm{d} D e p a r t m e n t}$ of Gastroenterology, Kitasato University School of Medicine, Sagamihara, Japan; eSchool of Medical Sciences, Universiti Sains Malaysia, Kota Bharu, Malaysia; ${ }^{f}$ Division of Gastroenterology and Hepatology, Department of Medicine, Nihon University School of Medicine, Tokyo, Japan

\section{Keywords}

Gastroesophageal junction · Diagnosis · Barrett esophagus .

Dysplasia $\cdot$ Adenocarcinoma

\begin{abstract}
Background: Epidemiology data of gastroesophageal junction (GEJ) cancers in Asia are extremely scarce. It is hardly registered by any cancer registry in the region, and only a few reports are available. Based on existing literature works, the overall trend indicates similar or gradually increasing GEJ cancers in Asia but comparably less than the West. The increasing trend in Asia is likely a result of rising risk factors, especially of gastroesophageal reflux disease and obesity. Summary: However, epidemiology data may be misleading due to several contentious diagnostic issues. The diagnostic conundrums are due to inherent complexity of the GEJ as a functional and pathological unit. Challenging diagnostic issues in Asia include the following: nonstandardized landmark of the GEJ, misclassification of Barrett esophagus, targeted versus nontargeted tissue sampling, histopathology disagreement and challenges in screening or surveillance of dysplastic BE and early GEJ cancer. The recent Asian-Pacific survey led by the Asian Barrett Consortium (ABC) has pro-
\end{abstract}

karger@karger.com

(c) 2021 S. Karger AG, Basel

www.karger.com/dig

Karger" vided useful insights into these contentious issues. A key learning point from these diagnostic limitations is that the awareness of the disease and adherence to existing recommendations or guidelines are poor in the region. Key Messages: Standardization in diagnostic methodology is vital for accurate epidemiology data, and this can only come from better awareness and adherence through educational and international efforts. Last, surveillance strategy may need a paradigm shift from a purely diagnostic approach to a combined targeted surveillance and treatment approach using novel endoscopic techniques.

(c) 2021 S. Karger AG, Basel

\section{Introduction}

Globally, the incidence of esophageal adenocarcinoma (EAC) is increasing [1]. It is widely known that the ageadjusted incidence rate of EAC is higher among Caucasians than Asians [2]. Most EACs occurred in the lower esophagus and at the gastroesophageal junction (GEJ). Although data are limited, GEJ cancer [3] is increasing in incidence in the Western population, and this is in contrast to the Asian population [4]. While the epidemiological differenc- 


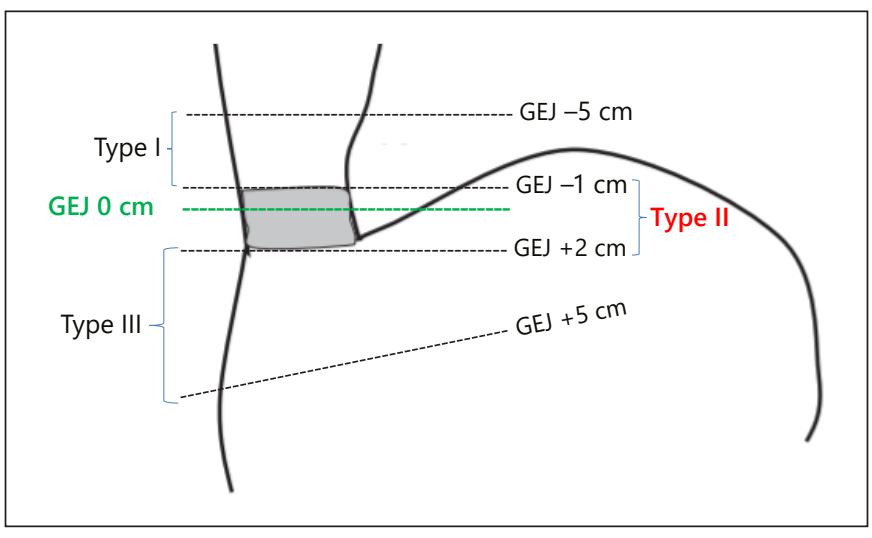

Fig. 1. Siewert-Stein classification of esophageal adenocarcinoma. Type I: adenocarcinoma of the distal esophagus $(-1$ to $-5 \mathrm{~cm}$ above GEJ). Type II: adenocarcinoma of the cardia $(-1 \mathrm{~cm}$ above to $+2 \mathrm{~cm}$ below GEJ). Type III: sub-cardial adenocarcinoma ( +2 to $+5 \mathrm{~cm}$ below GEJ). GEJ, gastroesophageal junction.

es of GEJ cancers between the East and West may have been true, diagnostic limitations inherent to the complex region of the GEJ may be another factor for the differences.

It is believed there are 2 biologically distinct forms of GEJ cancers. First, lesions above the GEJ are associated with gastroesophageal reflux disease (GERD) and Barrett esophagus (BE), that is, Siewert I (shown in Fig. 1). Second, lesions below the GEJ are associated with chronic atrophic gastritis and Helicobacter pylori infection and are histologically similar to gastric adenocarcinoma, that is, Siewert III $[5,6]$. Siewert II is a combination of the 2 biological forms of GEJ cancers. Despite the biology, clinical significance of defining the EAC origin from the esophagus and/or the gastric epithelium is unclear at the present moment. In particular, the distinction does not seem to affect management or the prognosis, but outcome data are limited [7].

While long-segment BE (LSBE) carries a higher annual incidence of progression to EAC than that of short-segment $\mathrm{BE}$ (SSBE), the risk of getting GEJ cancers did not reduce with SSBE. In Asia, SSBE is the predominant form of BE, whereas LSBE is found commonly among the Caucasians [8]. However, SSBE is often underdiagnosed or even missed, and the predominance of SSBE in Asia highlights an urgent need for standardization in the diagnosis of $\mathrm{BE}$ and GEJ cancers. In particular, an accurate system for diagnosing BE is needed to prevent misclassification and delay in preventive management. Standardization could also avoid overdiagnosis and unnecessary endoscopic surveillance, especially the ultrashort-segment BE (USSBE) of which the malignant potential is still unclear [2].
In the current review, we will first visit the epidemiology and the risk factors of GEJ cancers in Asia. Diagnostic conundrums will be reviewed next as its understanding is critical for the purpose of standardization and accuracy of epidemiological and research data.

\section{Methodology}

Literature search was conducted using databases including PubMed, Medline, and PMC, inclusive of all years until May 2021. All literatures were in the English language and consisted of original articles (systematic review or meta-analysis, randomized trials, and observational reports) and were available, conference proceedings. A recent Asia-Pacific survey led by the Asian Barrett Consortium $(\mathrm{ABC})$ has provided many insights into contentious issues discussed in the current review [9], and this may be potentially biased; however, it is also recognized that existing articles might be outdated and do not capture enough details unlike the ABC article. The search terms for databases included the following: gastroesophageal junction, adenocarcinoma, Barrett esophagus, epidemiology, dysplasia, diagnosis, sampling, and histology.

\section{Epidemiology and Risk Factors}

There are limited data on the incidence and trend of GEJ cancers in Asia since data are not captured by any existing cancer registry. Available trend studies are heterogeneous with some reported 2-3-fold increase and another reported similar over the years. For instance, in Japan, there are no data from the cancer registry other than a few published reports. The proportion of GEJ adenocarcinoma among operated advanced gastric adenocarcinoma was gradually increasing from $2.3 \%$ in $1962-1965$ to $10 \%$ in $2001-2005$. From this subset of patients, the proportion of Siewert II tumors had increased from $28.5 \%$ in $1962-1965$ to $57.3 \%$ in 2001. Interestingly, the proportion of Siewert I tumors had remained unchanged at $1 \%$ in the same time period, while Siewert III proportion has declined. The decline of Siewert III is likely attributed to a decline in $H$. pylori prevalence in Japan as a result of Westernization, national gastric cancer screening, and $H$. pylori eradication program [10]. More recent data from Japan reported an increasing EAC incidence in both sexes from 2002 to 2014 [11]. In Hong Kong, the precise location of EAC is lacking in the cancer registry. Based on single center data, 548 patients were diagnosed with GEJ cancers between 1981 and 2015, with an average annual incidence of 16 (range 11-26), and there were no signs of an increasing or a decreasing trend. Unpublished data from Malaysia indicated a 3-fold increase of GEJ cancers over 5 years (2011-2015), more among females and with an incidence rate of $0.5 / 100,000$ 
Fig. 2. a GEJ landmark identification by the distal margin of palisade vessels which also coincides with the proximal margin of gastric folds. b Inflammation causing difficult GEJ landmark identification by palisade vessels. Proximal margins of gastric folds were used instead. c Effect of breathing and air insufflation causing landmark variation in proximal margin of gastric folds in the same patient. (i) Deep inspiration and full insufflation. (ii) Full expiration and partial insufflation. GEJ, gastroesophageal junction.

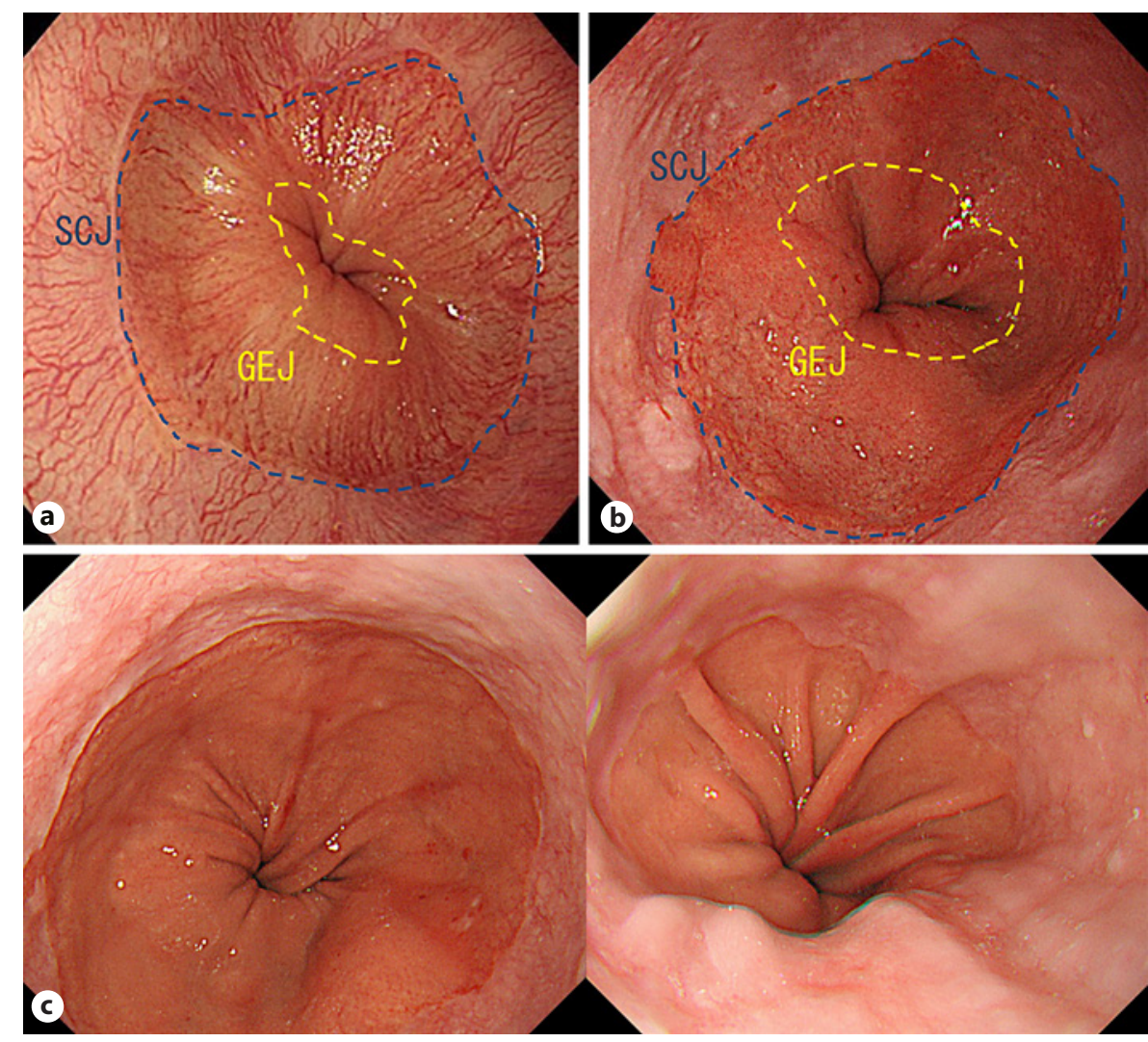

population. Half of GEJ cancers were Siewert II, and half were at an advanced stage upon presentation [5].

It was widely believed that GERD was almost unheard of in Asia until more recently. Despite a seemingly rising Asian GERD prevalence, the incidence of BE and GEJ cancers remained relatively similar or slightly increased over the years [12]. Abdominal obesity (visceral obesity) is a primary risk factor for the increasing incidence of $\mathrm{BE}$ and EAC in Asia, and most Asian patients have SSBE [8]. However, not all ethnics in Asia followed the same rules. For example, an exception was found in Japan, where there was a disproportionately higher prevalence of $\mathrm{BE}$ (up to $43 \%$ of patients undergoing upper endoscopy) in contrast to other Asian countries [13]. This could be due to inclusion of USSBE $(<1 \mathrm{~cm})$ in Japanese studies as opposed to other Asian countries. When USSBE was excluded from analyses, the prevalence in Japan was comparable to other Asian countries [8]. Excluding Japan, the prevalence of SSBE in patients undergoing gastroscopy in Asia ranged from 0.7 to $5.8 \%$, while the LSBE prevalence in Asia including Japan was $0.2-2.5 \%$ [14]. However, the prevalence of LGD, HGD, and EAC was $6.9 \%, 3.0 \%$, and $2.0 \%$, respectively, in histologically proven Asian BE [12].
Besides obesity, other risk factors for BE include $>5$ years of GERD symptoms, age $>50$ years, male sex, tobacco usage, presence of hiatus hernia, family history of $\mathrm{BE}$ (first-degree relative), and Caucasian race $[2,8]$. However, the risk factors for developing EAC or GEJ cancers include the presence of $\mathrm{BE}$, acid and/or bile reflux, old age, male sex, cigarette smoking, reduced fruit and vegetable intake, high red-meat consumption, family history, thoracic radiotherapy, and also Caucasian race $[1,15,16]$. The role of $H$. pylori infection as a risk factor is rather controversial. In Asia, $H$. pylori infection is more prevalent than in the West; however, recent prevalence is showing a widespread decline across Asia. While some studies have shown that $H$. pylori is protective against GERD and EAC in Asia, other observational studies did not $[2,17,18]$.

\section{Diagnostic Conundrums}

\section{Nonstandardized Landmark of the GEJ}

The very first and fundamental challenge is an accurate and accepted definition of the GEJ. Without a precise landmark acceptable to all, any epidemiological data will 
be variable, and any research data not comparable due to the inherent heterogeneity in methodology. Unfortunately, there is no universal definition of the GEJ at the present moment, but at least 2 definitions are more widely acceptable in Asia. The Japan Esophageal Society (JES) defines the lower margin of esophageal palisade vessels as the endoscopic landmark of the GEJ. However, inflammation, dysplastic changes, and the presence of nearby gastric longitudinal vessels might make identification difficult (shown in Fig. 2a, b). In such situation, the proximal margin of the longitudinal folds of the gastric greater curvature may be used instead [19]. American, British, Australian, and Asia-Pacific guidelines have recommended using proximal margin of the gastric folds to define the GEJ [2, 20-22]. This definition also produces a more consistent and reproducible landmark. However, excessive air deflation during gastroscopy, breathing movements, cardiac pulsations, lower esophageal sphincter contractions, and gastric mucosal atrophy might cause positional variations of the proximal margin of the gastric folds [23] (shown in Fig. 2c). Interestingly, from a recent survey led by the $\mathrm{ABC}$ to determine practices among AsiaPacific endoscopists, the squamocolumnar junction was the preferred landmark of the GEJ in $42 \%$, and only $19.5 \%$ preferred the proximal gastric folds [24]. Clearly, the squamocolumnar junction has a number of limitations including that it is frequently displaced by hiatus hernia, ulcers, or the columnar-lined epithelium. From a pathological standpoint, the concept to define the GEJ as a zone may be more relevant to the Siewert classification. A type II tumor is defined as cancer within $1 \mathrm{~cm}$ proximal to 2 $\mathrm{cm}$ distal to the GEJ. This "zone" correlates well with the histological presence of the cardiac gland, which is present with a high degree of frequency within this range of the Siewert definition [7]. Furthermore, in Siewert type II tumors, presence of cardiac glands and intestinal metaplasia (IM) were equally common [25].

\section{Different Classifications of $B E$}

For endoscopic grading of $\mathrm{BE}$, the Prague C \& M criterion, introduced in 2016, is a validated classification with high interobserver reliability (shown in Fig. 3). It is currently recommended by most guidelines. The definition of $\mathrm{BE}$ segments $<1 \mathrm{~cm}$ remains vague with some labeling it as the USSBE, while other guidelines have excluded them as a part of $\mathrm{BE}$ and attributed them as just an irregular Z-line. Distinguishing USSBE (length $<1 \mathrm{~cm}$ ) and the irregular Z-line remains subjective because of high interobserver variability in endoscopic findings [20, 26]. Clinical significance of USSBE remains unclear since

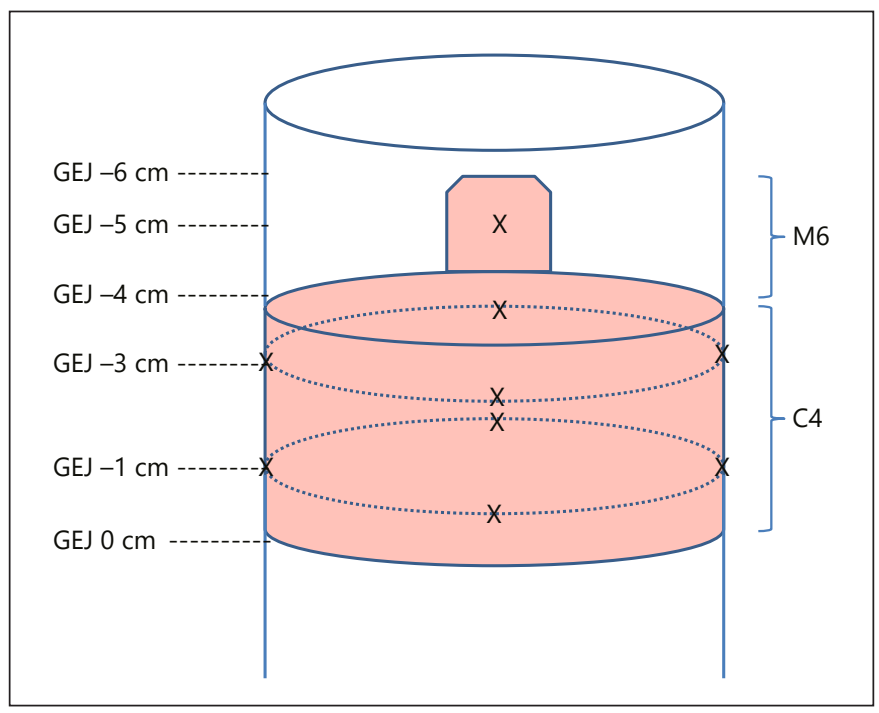

Fig. 3. Example of the Seattle biopsy protocol for BE (Prague classification C4M6). X, biopsy location; C, circular extent of BE; M, maximum extent of BE. BE; Barrett esophagus.

it appears that neither the risks of EAC nor dysplasia were higher [2]. Not all Asian guidelines or endoscopists adopt the Prague C \& M classification. In the recent Asia-Pacific survey, $46.1 \%$ of Japanese endoscopists claimed they had never used Prague C \& M compared to $15.8 \%$ outside Japan [9]. Even when Prague classification was used in Japan, the GEJ as defined in the Prague classification was not followed as only $12.6 \%$ of Japanese endoscopists adopted the proximal margin of gastric folds as the GEJ landmark [9]. In Japan, the JES defines LSBE as circular Barrett mucosa extending longitudinally for $\geq 3 \mathrm{~cm}$ (equivalent to the Prague Classification $\mathrm{C}$ component). Circular Barrett mucosa $\leq 3 \mathrm{~cm}$ in length or noncircular Barrett mucosa is classified as SSBE, even if the Prague Classification $\mathrm{M}$ component is $>3 \mathrm{~cm}$. However, the upper and lower limits of the SSBE length are not determined [8]. Other non-Japanese guidelines have classified $\mathrm{BE}$ based on the longest extent of the columnar epithelium (Prague Classification M component) $>3 \mathrm{~cm}$ as LSBE, with SSBE $>1 \mathrm{~cm}$ and $<3 \mathrm{~cm}$ in length [2, 20-22].

\section{Targeted or Nontargeted Tissue Sampling?}

Advances in technology have allowed conventional whitelight endoscopy using high-definition endoscopes with a video input and output of at least 1,280 × 1,024 pixels and with magnification capabilities of up to $45-200 \times$ as the standard of care in the assessment of BE and or dysplasia [2, 20, 21,27]. This can be augmented with advanced imaging techniques
Tan/Soh/Kusano/Lee/Gotoda 
such as narrow-band imaging (NBI), blue-light imaging, and chromoendoscopy to facilitate targeted biopsies and to improve dysplasia detection. At the moment, advanced imaging is not routinely recommended and is not superior to conventional high-definition systems [2, 20, 21]. The Barrett's International NBI Group (BING) criteria, which is based on the mucosal and vascular regularity, was shown to have an overall diagnostic accuracy of $85.4 \%$ to detect dysplasia in BE [28]. Acetoacetic acid chromoendoscopy using the Portsmouth acetic acid classification is a validated system to endoscopically differentiate nondysplastic $\mathrm{BE}$ (NDBE), dysplastic $\mathrm{BE}$, and Barrett's adenocarcinoma as well as to assist targeted biopsies [29]. The Seattle biopsy protocol is the recommended approach for obtaining nontargeted biopsies (shown in Fig. 3). [22, 30] This is a systematic biopsy protocol beginning from the GEJ, of 4 quadrants at $1-2 \mathrm{~cm}$ intervals. Based on a Chinese study, this was shown to outperform NBI guided biopsies in detecting IM and dysplasia in Chinese patients [31]. However, other studies using NBI have shown that NBItargeted biopsies had the same detection rate for IM as highdefinition white-light examination and Seattle protocol and that NBI-targeted biopsies also detected more areas with dysplasia [32]. Unfortunately, compliance to the Seattle protocol for BE biopsies remains poor at $6.3 \%$ in the Asia-Pacific region [9]. In contrast, Seattle protocol adherence was $51.2 \%$ in USA, were SSBE had a better protocol compliance than that of LSBE [33]. Could this be due to it being a cumbersome biopsy protocol or the more widespread use of NBI and other imaging techniques in Asia? Furthermore, it remains unknown if targeted versus nontargeted biopsies could translate into higher rates of missed dysplasia or early GEJ cancer. Other novel diagnostic methods have included Raman spectroscopy and artificial intelligence which showed promising results to differentiate dysplastic BE or early GEJ cancers from NDBE [34].

\section{Histopathology of BE, Dysplasia, and Early GEJ Cancer}

The controversy lies in the requirement of IM and/or goblet cells to diagnose BE. This criterion is not a prerequisite in the JES, Asia-Pacific, and British guidelines [8, $20,21]$, while in the American, European, and Australian guidelines, it is a requirement $[2,23,31]$. In addition, the terms high-grade dysplasia (HGD) outside of Japan and intramucosal carcinoma within Japan often create ambiguity $[2,8]$. The reason IM is required in diagnosis is due to a higher risk of developing EAC when IM was present in biopsies, although EAC can also develop without prior IM [35]. On the other hand, patchy involvement of IM within areas of the columnar epithelium might cause Se-

Gastroesophageal Junction Cancer

Diagnostic Conundrums attle protocol-based biopsy sampling to miss affected areas containing IM. This can be partially compensated by taking up to 8 biopsy samples in order to improve the diagnostic yield of IM $[2,8]$. Diagnosis of low-grade dysplasia (LGD) can be particularly challenging even for expert histopathologists. The presence of LGD and HGD should preferably be confirmed by expert gastrointestinal pathologists prior to any ablative treatment or resection [30]. Guidelines recommend 2 histopathologists to report on dysplasia, especially in specimens "indefinite for dysplasia" due to common intraobserver and interobserver variability even among experts. In situations where a diagnosis is indefinite, a repeat biopsy after 6 months is warranted $[2,20,34]$. However, in resource-limited centers in Asia, a lack of access to gastrointestinal histopathologists may pose difficulty in implementing this requirement. Due to difficulty in assessment of dysplasia, several novel biomarker techniques have emerged with promising results. These include tumor protein p53 immunohistochemistry, DNA analysis for aneuploidy and tetraploidy, cyclin A, cyclin D1, and fluorescence in situ hybridization techniques. At present, molecular biomarkers do not have sufficient evidence to supplement or supplant endoscopy and histopathology outside of a research setting [35].

\section{Screening or Surveillance of BE, Dysplasia, and Early GEJ Cancer}

Screening aims to detect dysplastic BE and early GEJ cancer with the hope for EAC prevention and improving patient survival. Among patients with EAC, $>90 \%$ do not have a history of prior $\mathrm{BE}$, and $>40 \%$ do not have a prior diagnosis of GERD [16]. In addition, the rate of progression from NDBE through LGD to HGD to GEJ cancer is unknown, although progression is likely more rapid with HGD than other forms of BE [36]. Current data suggest a better survival outcome if EAC is diagnosed in preexisting $\mathrm{BE}$ than in its absence [37]. The unknown natural history, a low prevalence of BE and GEJ cancers, and limited resources in Asia have made endoscopy screening not cost-effective, unless these countries have an existing screening program for gastric cancer (e.g., Japan and Korea) [20]. Based on clinical observations, dysplastic or neoplastic lesions in SSBE were better recognized than in LSBE, and nonvisible dysplastic lesions in SSBE were usually regenerative mucosa. As such, surveillance with random biopsies may not be practical, and targeted biopsies followed by endoscopic resection may be more useful.

The current guideline from the Asia-Pacific does not recommend routine surveillance for NDBE as there is no 

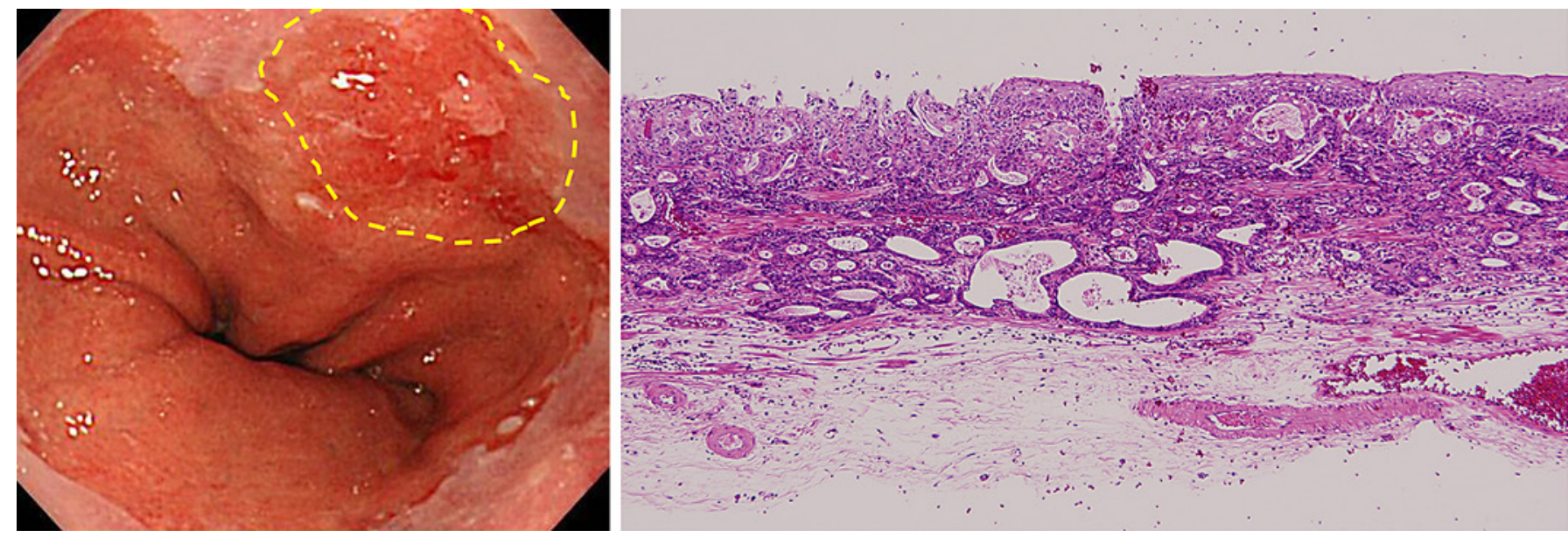

Fig. 4. Early GEJ adenocarcinoma resected by ESD with histopathological examination showing muscularis mucosa and submucosal invasion by high-grade dysplastic epithelial cells in a short-segment Barrett specimen resected by ESD. Both vertical and horizontal margins are clear, and R0 resection was achieved endoscopically. ESD, endoscopic submucosal dissection; GEJ, gastroesophageal junction.

proven benefit [21]. The incidence in progression of nondysplastic SSBE to EAC (0.07\%/year) is lower than that of LSBE (0.25\%/year) [38]. Thus, some authors in Asia have recommended a 3-year surveillance interval for nondysplastic LSBE and a 5-year interval for nondysplastic SSBE [39]. In contrast, the Western guidelines recommend routine 3-5 year intervals for surveillance of NDBE, depending on the length of $\mathrm{BE}$; however, no randomized controlled trials have proven benefit of such approach [40, 41]. From the Australian perspective, there is recommendation for closer surveillance of higher risk NDBE patients such as those with LSBE, males, smokers, and older agegroups [22]. There are limited Asian data on progression of NDBE to dysplastic lesions or EAC on surveillance. In a small study in Taiwan on patients with NDBE $(n=51)$, the LGD progression incidence was $2.9 \%$ per year, with $0 \%$ for HGD and $0.4 \%$ for EAC [42]. Targeted surveillance based on presence of dysplastic BE is the current recommendation for Asia, with a weaker recommendation of 3-5 year surveillance intervals using endoscopy with biopsy (Seattle protocol or targeted biopsies) for NDBE due to a lack of randomized controlled trial-proven benefits [21]. Interestingly, Hojo et al. [14] demonstrated that Asian endoscopists tend to survey BE patients with or without dysplasia with intervals ranging from 3 months to $>3$ years, with LSBE having closer intervals. When dealing with dysplastic BE, histologically confirmed LGD can be managed by either surveillance at 6 months or resection with or without ablation. Lesions with HGD and early EAC need to be resected or ablated $[2,19,20,28]$.
With the advent of novel endoscopic resection techniques including endoscopic submucosal resection (ESD), targeted surveillance of early GEJ cancers may be potentially combined with endoscopic resection (shown in Fig. 4). From the survey, for BE without a lesion but where biopsies showed HGD, a large majority of Japanese endoscopists and about half outside of Japan would go for ESD [9]. At the moment, the combination strategy does not work because of limited adoption of ESD outside Japan, but this may change in the future with better robotic technology and better techniques that are being developed. There is a possibility that ESD for early GEJ cancers will be more widely adopted across Asia in the near future, similar to the success of ESD for early gastric cancer in Japan. In addition, clinical trial is needed to clarify if this combination strategy will improve the clinical outcome. Until then, the combination strategy as suggested here is at the moment an opinion and not supported by evidence.

\section{Conclusion}

Available epidemiology data on GEJ cancers are extremely limited. It is also not captured by the existing cancer registry of Asian countries. The overall trend indicates similar or gradually increasing GEJ cancers in Asia, but comparably less than the West. However, epidemiology data may be misleading due to contentious diagnostic issues. These include a nonstandardized land- 
mark of the GEJ, classification of BE, tissue sampling, histopathology and screening or surveillance of dysplastic BE, and early GEJ cancer. The recent Asian-Pacific survey led by the $\mathrm{ABC}$ has provided insights into these contentious issues. A key point from the survey is that awareness and adherence to existing recommendations are poor in the region. Standardization in the methodology is vital, and this can only come from better awareness and adherence through educational and international efforts. Last, surveillance strategy needs a paradigm shift from a purely diagnostic approach to a combined targeted surveillance and treatment approach through novel endoscopic techniques.

\section{Conflict of Interest Statement}

Takuji Gotoda is an associate editor of Digestion. Other authors have no conflicts of interest to declare.

\section{Funding Sources}

None of the authors have any funding sources to declare.

\section{Author Contributions}

Y.Y.L. contributed to conceptualization, technical guidance, review, and editing. P.O.T. contributed to original draft, figures, and editing. A.Y.S.S. contributed to review and editing. C.K. contributed to review and editing. T.G. contributed to review, photographs, and editing.

\section{References}

1 Pakzad R, Mohammadian-Hafshejani A, Khosravi B, Soltani S, Pakzad I, Mohammadian $\mathrm{M}$, et al. The incidence and mortality of esophageal cancer and their relationship to development in Asia. Ann Transl Med. 2016; $4(2): 29$.

2 Shaheen NJ, Falk GW, Iyer PG, Gerson LB; American College of Gastroenterology. ACG clinical guideline: diagnosis and management of Barrett's esophagus. Am J Gastroenterol. 2016;111(1):30-51. Erratum in: Am J Gastroenterol. 2016 Jul;111(7):1077.

3 Rüdiger Siewert J, Feith M, Werner M, Stein HJ. Adenocarcinoma of the esophagogastric junction: results of surgical therapy based on anatomical/topographic classification in 1,002 consecutive patients. Ann Surg. 2000; 232(3):353-61.

4 Lu CL, Lang HC, Luo JC, Liu CC, Lin HC, Chang FY, et al. Increasing trend of the incidence of esophageal squamous cell carcinoma, but not adenocarcinoma, in Taiwan. Cancer Causes Control. 2010;21(2):269-74.

5 Hatta W, Tong D, Lee YY, Ichihara S, Uedo N, Gotoda T. Different time trend and management of esophagogastric junction adenocarcinoma in three Asian countries. Dig Endosc. 2017;29(Suppl 2):18-25.

6 Uedo N, Yoshio T, Yoshinaga S, Takeuchi M, Hatta W, Yano T, et al. Endoscopic gastric mucosal atrophy distinguishes the characteristics of superficial esophagogastric junction adenocarcinoma. Dig Endosc. 2017;29(Suppl 2):26-36. Erratum in: Dig Endosc. 2018 Jul; 30(4):557.

7 Ichihara S, Uedo N, Gotoda T. Considering the esophagogastric junction as a "zone." Dig Endosc. 2017;29 Suppl 2(Suppl 2):3-10.

8 Lee HS, Jeon SW. Barrett esophagus in Asia: same disease with different pattern. Clin Endosc. 2014;47(1):15-22.
9 Kew GS, Soh AYS, Lee YY, Gotoda T, Li YQ, Zhang Y, et al. Multinational survey on the preferred approach to management of Barrett's esophagus in the Asia-Pacific region. World J Gastrointest Oncol. 2021;13(4):279_ 94.

10 Kusano C, Gotoda T, Khor CJ, Katai H, Kato $\mathrm{H}$, Taniguchi $\mathrm{H}$, et al. Changing trends in the proportion of adenocarcinoma of the esophagogastric junction in a large tertiary referral center in Japan. J Gastroenterol Hepatol. 2008;23(11):1662-5.

11 Matsuno K, Ishihara R, Ohmori M, Iwagami $\mathrm{H}$, Shichijyo S, Maekawa A, et al. Time trends in the incidence of esophageal adenocarcinoma, gastric adenocarcinoma, and superficial esophagogastric junction adenocarcinoma. J Gastroenterol. 2019 Sep;54(9):784-91.

12 Shiota S, Singh S, Anshasi A, El-Serag HB. Prevalence of Barrett's Esophagus in Asian countries: a systematic review and meta-analysis. Clin Gastroenterol Hepatol. 2015;13(11): 1907-18.

13 Akiyama $\mathrm{T}$, Inamori $\mathrm{M}$, Akimoto $\mathrm{K}$, Iida $\mathrm{H}$, Mawatari $\mathrm{H}$, Endo H, et al. Risk factors for the progression of endoscopic Barrett's epithelium in Japan: a multivariate analysis based on the Prague C \& M Criteria. Dig Dis Sci. 2009; 54(8):1702-7.

14 Hojo M, Nagahara A, Hahm KB, Iwakiri R, Watanabe T, Rani AA, et al. Management of gastroesophageal reflux disease in Asian countries: results of a questionnaire survey. Digestion. 2020;101(Suppl 1):66-79.

15 Smyth EC, Lagergren J, Fitzgerald RC, Lordick F, Shah MA, Lagergren P, et al. Oesophageal cancer. Nat Rev Dis Primers. 2017; 3:17048. Published 2017 Jul 27.

16 Eluri S, Shaheen NJ. Barrett's esophagus: diagnosis and management. Gastrointest Endosc. 2017;85(5):889-903.
17 Lee YY, Raj SM, Sharif SE, Salleh R, Ayub MC, Graham DY. Incidence of esophageal carcinoma among Malays in North-Eastern Peninsular Malaysia: an area with an exceptionally low prevalence of Helicobacter pylori infection. Dig Dis Sci. 2011;56(5):1438-43.

18 Xue Y, Zhou LY, Lin SR, Hou XH, Li ZS, Chen $\mathrm{MH}$, et al. The effect of Helicobacter pylori eradication in patients with gastroesophageal reflux disease: a meta-analysis of randomized controlled studies. Dig Dis. 2020;38(4):261-8.

19 Fitzgerald RC, di Pietro M, Ragunath K, Ang Y, Kang JY, Watson P, et al. British Society of Gastroenterology guidelines on the diagnosis and management of Barrett's oesophagus. Gut. 2014;63(1):7-42.

20 Fock KM, Talley N, Goh KL, Sugano K, Katelaris P, Holtmann G, et al. Asia-Pacific consensus on the management of gastro-oesophageal reflux disease: an update focusing on refractory reflux disease and Barrett's oesophagus. Gut. 2016;65(9):1402-15.

21 Whiteman DC, Appleyard M, Bahin FF, Bobryshev YV, Bourke MJ, Brown I, et al. Australian clinical practice guidelines for the diagnosis and management of Barrett's esophagus and early esophageal adenocarcinoma. J Gastroenterol Hepatol. 2015;30(5):804-20.

22 Amano Y, Ishimura N, Furuta K, Takahashi Y, Chinuki D, Mishima Y, et al. Which landmark results in a more consistent diagnosis of Barrett's esophagus, the gastric folds or the palisade vessels? Gastrointest Endosc. 2006; 64(2):206-11.

23 Nunobe S, Nakanishi Y, Taniguchi H, Sasako M, Sano T, Kato H, et al. Two distinct pathways of tumorigenesis of adenocarcinomas of the esophagogastric junction, related or unrelated to intestinal metaplasia. Pathol Int. 2007;57:315-21. 
24 Sharma P, Dent J, Armstrong D, Bergman JJ, Gossner L, Hoshihara Y, et al. The development and validation of an endoscopic grading system for Barrett's esophagus: the Prague C \& M criteria. Gastroenterology. 2006;131(5): 1392-9.

25 Sharma P, Bergman JJ, Goda K, Kato M, Messmann H, Alsop BR, et al. Development and validation of a classification system to identify high-grade dysplasia and esophageal adenocarcinoma in Barrett's esophagus using narrow-band imaging. Gastroenterology. 2016;150(3):591-8.

26 Kandiah K, Chedgy FJQ, Subramaniam S, Longcroft-Wheaton G, Bassett P, Repici A, et al. International development and validation of a classification system for the identification of Barrett's neoplasia using acetic acid chromoendoscopy: the Portsmouth acetic acid classification (PREDICT). Gut. 2018;67(12): 2085-91.

27 Wang KK, Okoro N, Prasad G, WongKeeSong M, Buttar NS, Tian J. Endoscopic evaluation and advanced imaging of Barrett's esophagus. Gastrointest Endosc Clin N Am. 2011 Jan;21(1):39-51.

28 Weusten B, Bisschops R, Coron E, Dinis-Ribeiro M, Dumonceau JM, Esteban JM, et al. Endoscopic management of Barrett's esophagus: European Society of Gastrointestinal Endoscopy (ESGE) position statement. Endoscopy. 2017;49(2):191-8.
29 Lee SW, Lien HC, Chang CS, Lin MX, Chang $\mathrm{CH}$, Ko CW. Benefits of the Seattle biopsy protocol in the diagnosis of Barrett's esophagus in a Chinese population. World J Clin Cases. 2018;6(14):753-8.

30 Sharma P, Hawes RH, Bansal A, Gupta N, Curvers W, Rastogi A, et al. Standard endoscopy with random biopsies versus narrow band imaging targeted biopsies in Barrett's oesophagus: a prospective, international, randomised controlled trial. Gut. 2013;62(1):1521.

31 Soh YSA, Lee YY, Gotoda T, Sharma P, Ho KY; Asian Barrett's Consortium. Challenges to diagnostic standardization of Barrett's esophagus in Asia. Dig Endosc. 2019;31(6): 609-18.

32 Varghese S, Lao-Sirieix P, Fitzgerald RC. Identification and clinical implementation of biomarkers for Barrett's esophagus. Gastroenterology. 2012;142(3):435-41.e2.

33 Hamade N, Vennelaganti S, Parasa S, Vennalaganti P, Gaddam S, Spaander MCW, et al. Lower annual rate of progression of shortsegment vs long-segment Barrett's Esophagus to Esophageal adenocarcinoma. Clin Gastroenterol Hepatol. 2019;17(5):864-8.

34 Rastogi A, Puli S, El-Serag HB, Bansal A, Wani S, Sharma P. Incidence of esophageal adenocarcinoma in patients with Barrett's esophagus and high-grade dysplasia: a metaanalysis. Gastrointest Endosc. 2008;67(3): 394-8.

35 Tramontano AC, Sheehan DF, Yeh JM, Kong CY, Dowling EC, Rubenstein JH, et al. The impact of a prior diagnosis of Barrett's Esophagus on Esophageal adenocarcinoma survival. Am J Gastroenterol. 2017;112(8):1256-64.
36 Japan Esophageal Society. Japanese classification of Esophageal cancer, 11th edition: part II and III. Esophagus. 2017;14(1):37-65.

37 Amano Y, Kinoshita Y. Barrett Esophagus: perspectives on its diagnosis and management in Asian populations. Gastroenterol Hepatol. 2008;4(1):45-53.

38 Qumseya B, Qumseya B, Sultan S, Bain P, Jamil L, Jacobson B, et al. ASGE guideline on screening and surveillance of Barrett's esophagus. Gastrointest Endosc. 2019;90(3):33559.e2.

39 Weusten B, Bisschops R, Coron E, Dinis-Ribeiro M, Dumonceau JM, Esteban JM, et al. Endoscopic management of Barrett's esophagus: European Society of Gastrointestinal Endoscopy (ESGE) position statement. Endoscopy. 2017;49(2):191-8.

40 Lee SW, Lien HC, Peng YC, Lin MX, Ko CW, Chang CS. The incidence of esophageal cancer and dysplasia in a Chinese population with nondysplastic Barrett's esophagus. JGH Open. 2018 Aug 9;2(5):214-6.

41 Di J, Sharma N, Pérez LKM, Zhang J, Ho KY. Surveillance strategy of Barrett's esophagus in the Asian region with particular reference to its locoregional epidemiology. JGH Open. 2020 May 12;4(4):565-8.

42 Abrams JA, Kapel RC, Lindberg GM, Saboorian MH, Genta RM, Neugut AI, et al. Adherence to biopsy guidelines for Barrett's esophagus surveillance in the community setting in the United States. Clin Gastroenterol Hepatol. 2009 Jul;7(7):736-10; quiz 710. 